\title{
Europe has to step up its efforts to produce innovative and safe diabetes technology
}

\author{
Miriam Cnop ${ }^{1,2,3}$ - Tomasz Klupa ${ }^{3,4}$ - Nikolaos Tentolouris ${ }^{3,5}$ - Anna Novials ${ }^{3,6,7}$. \\ Rémy Burcelin $^{3,8} \cdot$ Mischa van Eimeren ${ }^{9}$
}

Received: 25 August 2017 / Accepted: 30 August 2017 /Published online: 24 September 2017

(C) Springer-Verlag GmbH Germany 2017

Keywords Artificial pancreas · Closed loop · European Database on Medical Devices · European parliament · Glucose meter $\cdot$ Innovation $\cdot$ Insulin pump $\cdot$ Legislation $\cdot$ Medical device $\cdot$ Safety

\begin{tabular}{ll}
\multicolumn{2}{l}{ Abbreviations } \\
EU & European Union \\
EUDAMED & European Database on Medical Devices \\
IDF & International Diabetes Federation
\end{tabular}

Miriam Cnop

mcnop@ulb.ac.be

1 ULB Center for Diabetes Research, Université Libre de Bruxelles, Route de Lennik 808 CP-618, 1070 Brussels, Belgium

2 Division of Endocrinology, Erasmus Hospital, Brussels, Belgium

3 EASD-EU Committee, EASD, Düsseldorf, Germany

4 Department of Metabolic Diseases, Jagiellonian University Medical College, Kraków, Poland

5 First Department of Internal Medicine, National and Kapodistrian University of Athens, Athens, Greece

6 Diabetes and Obesity Research Laboratory, Institut D'Investigacions Biomèdiques (IDIBAPS), Hospital Clinic de Barcelona, Barcelona, Spain

7 Centro de Investigación Biomédica en Red de Diabetes y Enfermedades Metabólicas Asociadas (CIBERDEM), Spain, http://www.ciberdem.org

8 Institut des Maladies Métaboliques et Cardiovasculaires (I2MC), Inserm 1048, Toulouse, France

9 EASD Headquarters, Düsseldorf, Germany
To the Editor: There is an ongoing (legislative) debate on medical devices and in vitro diagnostics at the European Union (EU) level that will influence the future of diabetes technology and, in particular, the safety and innovation of new devices.

Diabetes technology, such as insulin pumps and glucose meters, has experienced major advances over recent decades. This has changed their use from being termed 'sporadic' in the 1970s to now being widespread, and has led to significantly improved management and care of people with diabetes [1]. There are grounds for concern, however, about the safety of these increasingly complex devices. A relevant example is the 'closed loop system' or 'artificial pancreas' that will be available in Europe approximately 1 year from now. This system enables insulin pumps to automatically administer insulin with only limited intervention by patients. This is a breakthrough in diabetes care and has the potential to significantly improve the daily lives and long-term health of people with diabetes. However, its (semi) automatic character raises safety concerns, particularly in view of past and more recent research on the general performance of insulin pumps.

In 2009, Guilhem and colleagues reported that $36 \%$ of randomly selected pumps failed partially or completely after a median time of 15 months [2]. A recently published update confirmed that pump failure continues to be a common problem [3].

Unfortunately, the full scale of the problem remains unknown as there is only limited access to vigilance data. The EASD has monitored these developments and, in 2012, it added the safety of diabetes technology to its key strategic priorities. At around the same time, the European Parliament and the European Council started negotiating new legislation in this area following diverging interpretation of the current European directives and the fraudulent production of Poly Implant Prothèse (PIP) silicone breast implants in 2010 [4], 
which highlighted weaknesses in the current legislative framework.

In statements (most notably a joint statement by the EASD and the ADA Diabetes Technology Working Group [5]), at conferences and during meetings with relevant stakeholders, the EASD has called upon the EU decision makers, industry and other stakeholders to work together towards safer diabetes technology. One of its focal points is the improved scrutiny for insulin pumps before and after they enter the European market.

Despite the recent adoption of legislation [6], the debate will continue. The coming 3 to 5 -year implementation period entails the drafting of over 80 implementing or delegating acts that will provide answers to open questions in the legislative text, such as the level of public access to the soon-to-be refurbished European Database on Medical Devices (EUDAMED), the exact requirements for clinical evidence and the final conformity assessment procedures.

It is therefore key that the diabetes community continues to follow this topic. It must ensure that the final legislative text stimulates innovation while guaranteeing the highest levels of safety.

The EASD-EU Committee has recently highlighted the legislative questions around diabetes technology at a high level conference in the European Parliament, hosted by a member of the European Parliament, Bogdan Wenta [7]. The event, held on 31 May 2017, brought together a panel of speakers that included people with diabetes, academics, policy makers, politicians and industry representatives. In addition to the new legislation, innovation was one of the key topics of the conference.

From the event, it became clear that Europe and the USA are competing in the field of innovation of diabetes technology and medical devices, and in vitro diagnostics in general. Despite recent cuts in the US research budget, Europe is likely to remain at a disadvantage in this field in the short term. An example is the recent commercialisation of the artificial pancreas in the USA, while this technology is not expected to enter the European market prior to 1 year from now.

It is important that the latest innovations in diabetes technology can be accessed not just by the lucky few, but by all European people with diabetes. Cristina Petrut, board member of International Diabetes Federation (IDF) Europe, formulated this perfectly during the meeting by stating that "nothing is more frustrating for a patient to know there is a device out there that can significantly improve your daily life, but you are unable to obtain it'.

Further to promoting equitable access by means of coherent and transparent legislation, a number of other tools were mentioned during the event that could stimulate innovation, including increased efforts to bring researchers in this field together, a new scientific journal dedicated to this topic and increasing the number and resources of European academic centres focusing on diabetes (technology).

This meeting demonstrated both the differences between the different stakeholders and their strong commitment to collaboration. Presentations by Professor Andrew Boulton, chair of the Alliance for European Diabetes Research (EURADIA), and Professor Sehnaz Karadeniz, President of IDF Europe, showed that broad access to EUDAMED, strict guidelines for clinical research, and pre-market tests for efficacy and safety are the main priorities for these organisations during the upcoming implementation of the legislation. The EASD supports these strategic priorities and calls upon all members of its community to discuss them with their peers, as well as with national and European politicians and policy makers.

Funding Work in the authors' laboratories is supported by the European Union's Horizon 2020 research and innovation programme, project T2DSystems, under grant agreement no. 667191, and the Innovative Medicines Initiative 2 Joint Undertaking, project INNODIA, under grant agreement no. 115797, which receives support from the European Union's Horizon 2020 research and innovation programme, EFPIA, JDRF and the Leona M. and Harry B. Helmsley Charitable Trust.

Duality of interest The authors declare that there is no duality of interest associated with this manuscript.

Contribution statement $\mathrm{MC}$ and MvE wrote the paper. TK, NT, AN and RB revised it. All authors approved the final version.

\section{References}

1. Thabit H, Hovorka R (2016) Continuous subcutaneous insulin infusion therapy and multiple daily insulin injections in type 1 diabetes mellitus: a comparative overview and future horizons. Expert Opin Drug Deliv 13:389-400

2. Guilhem I, Balkau B, Lecordier F et al (2009) Insulin pump failures are still frequent: a prospective study over 6 years from 2001 to 2007. Diabetologia 52:2662-2664

3. Guenego A, Bouzille G, Breitel S et al (2016) Insulin pump failures: has there been an improvement? Update of a prospective observational study. Diabetes Technol Ther 18:820-824

4. Wikipedia (2017). Poly Implant Prothèse. Available from https://goo. gl/vJfHPi. Accessed 17 Aug 2017

5. Heinemann L, Fleming GA, Petrie JR, Holl RW, Bergenstal RM, Peters AL (2015) Insulin pump risks and benefits: a clinical appraisal of pump safety standards, adverse event reporting and research needs. A joint statement of the European Association for the Study of Diabetes and the American Diabetes Association Diabetes Technology Working Group. Diabetologia 58:862-870

6. European Commission (2017). Medical devices regulatory framework. Available from https://ec.europa.eu/growth/sectors/medicaldevices/regulatory-framework en. Accessed 17 Aug 2017

7. European Association for the Study of Diabetes (2017). Diabetes technology meeting report. Available from https://www.easd.org/ sites/default/files/EASD\%20EU\%20Technology\%20Report final. pdf. Accessed 17 Aug 2017 